\title{
Magnetic confinement and the Linde problem
}

\author{
Yu.A.Simonov, \\ State Research Center \\ Institute of Theoretical and Experimental Physics \\ 117218, Moscow, B.Cheremushkinskaya 25, Russia
}

May 24, 2017

\begin{abstract}
Perturbation theory of thermodynamic potentials in QCD at $T>T_{c}$ is considered with the nonperturbative background vacuum taken into account. It is shown that the colormagnetic confinement in the $\mathrm{QCD}$ vacuum prevents the infrared catastrophe of the perturbation theory present in the case of the free vacuum (the "Linde problem"). A short discussion is given of the applicability of the nonperturbative formalism at large $T$ and of the relation with the HTL theory. The observation of A.D.Linde, that the terms $O\left(g^{n}\right), n>6$ contribute to the order $O\left(g^{6}\right)$ is confirmed also with the account of the colormagnetic confinement, and it is shown that the latter makes these terms IR convergent, and summable. As a result one obtains the selfconsistent theory of the gluon plasma.
\end{abstract}




\section{Introduction}

The Linde problem in the thermal QCD has a long history, see [1, 2] and refs therein. It has occurred in the thermal perturbative QCD, where similarly to QED, the originally massless constituents (gluons) acquire effective perturbative mass operators $m(T)$, which regulate the convergence of $g^{n}$ terms and of the whole perturbative series. Correspondingly, the colorelectric screening mass $m_{D}(T)$, obtained from $\Pi_{00}(T)$ (similarly to the QED case) starts from $g T$, however the colormagnetic screening mass does not exist perturbatively [1, 2] (again, as in QED), and if introduced effectively as $O\left(g^{2} T\right)$, the perturbative series is not defined at the order $g^{6}$ (problem (1)). Linde also remarks, that the higher order diagrams contribute to the same order (problem (2)).

Meanwhile the effective perturbative theory of thermal QCD (the hard-thermalloop (HTL) theory) was developed in [3, 4, using the colorelectric $m_{D}(T)$ and the resummation technic through order $g^{2}, g^{3}, g^{4}, g^{5}$, which appears to be quite successful, see [5] for a review. The nonperturbative nature of the magnetic scale $g^{2} T$, which appears necessarily at $O\left(g^{6}\right)$ can be connected to the 3d Yang Mills theory, see e.g. [6].

A natural question arises, how this situation can be explained and treated in a 4d approach to QCD, where nonperturbative (np) physics (including confinement) is taken into account?

In what follows we shall consider the np approach to QCD, developed in [7, 8, 9, 10, 11, 12]. For an alternative approach see [13, 14].

Most effects in QCD at low and intermediate energies cannot be explained without np dynamics, which enters in the theory, e.g. via the string tension $\sigma$, or a mass of some meson $(\rho, K)$, or else the constant $\Lambda_{Q C D}$, entering in $\alpha_{s}(Q)$. The approach of the np vacuum, ensuring confinement, and stabilizing perturbation theory, was developed in [7 for QCD at zero temperature $T$, and in [8, 9, for $T>0$, see reviews [10, 11] for $T=0$ and [12] for $T>0$. The problem of the confinement and deconfinement is treated in our approach, called the Field Correlator method (FCM), taking into account two kinds of colorelectric correlators $D^{E}(z), D_{1}^{E}(z) \sim\left\langle E_{i}(x) E_{i}(y)\right\rangle$, and two kinds of colormagnetic, $D^{H}(z), D_{1}^{H}(z) \sim\left\langle H_{i}(x) H_{i}(y)\right\rangle$, where the first ones $\left(D^{E}, D^{B}\right)$ are of purely nonabelian character, while $D_{1}^{E}, D_{1}^{H}$ exist also in QED.

Assumed in [8] and later confirmed on the lattice [15], that the nonabelian colorelectric correlator $D^{E}(z)$ vanishes together with confinement at $T_{c}$, while all others stay nonzero for $T>T_{c}$, in particular the nonabelian colorelectric correlator $D_{1}^{E}$, responsible for the nonzero Polyakov lines, while the nonabelian colormagnetic correlator $D^{H}(z)$ ensures the magnetic confinement for the motion in the spatial planes. 
This property was studied in the FCM formalism in [16], and analytically and numerically in a different approach in [17, see also [18] for later developments.

As a result of magnetic confinement there appears the spatial string tension, which defines the area law of the spatial projection of any Wilson loop in $4 \mathrm{~d}$,

$$
\langle W(C)\rangle=\exp \left(-\sigma_{s} A_{3 d}(C)\right), \quad \sigma_{s}=\frac{1}{2} \int d^{2} z D^{H}(z) .
$$

Moreover, $D^{H}(z)$ can be calculated via the gluelumps [19], known both analytically [20] and on the lattice [21], which yields the relation

$$
\sqrt{\sigma_{s}(T)}=c_{\sigma} g^{2}(T) T
$$

where $c_{\sigma}$ is of the np origin, as shown in the appendix. This coincides with the lattice data results [22], where $c_{\sigma}=0.566 \pm 0.013$.

The two-loop approximation is generally used for $g^{2}(T)$ [23].

One can now consider any QCD diagram and the whole perturbative series as being immersed in the np vacuum, so that all closed loops in the $3 \mathrm{~d}$ space are covered by the confining film, while for $4 \mathrm{~d}$ loops covered is its $3 \mathrm{~d}$ projection. This fact turns over the whole thermal QCD dynamics.

Namely, as we show below, the spatial Wilson loops not only serve as a cut-off factor at the distance $X_{\max } \sim \frac{1}{\sqrt{\sigma_{s}}}$, but due to Eq. (2) this cut-off depends on $g(T)$ and converts the perturbative $O\left(g^{n}\right)$ term into $O\left(g^{6}\right)$. (Problem 2 of Linde). Exactly the same situation would occur, if instead of spatial confinement one introduces the magnetic mass of gluons.

Then again Eq. (2) implies that the magnetic mass $m_{D}^{H} \sim \sqrt{\sigma_{s}}$ as a cutoff parameter makes the 4 loop integral convergent, which resolves, what can be called the problem 1 of Linde, as will be clarified below. This is purely np result, irrespectively of the appearing $g^{2}$ factor. The problem (2) of Linde (see (iii) in [1] on p. 290) that the sum of the infinite ladder of gluon loops with $n>4$ contributes to the same order $g^{6} T^{4}$ also occurs in this case of magnetic mass.

However, the notion of magnetic mass (or any other effective mass) is irrelevant in the case of confinement, since gluons are connected by the confining string, which constitutes the most part of the total energy (mass) of the system in contrast to the free motion of a gluon with any effective mass.

Coming back to the results of the perturbation theory and comparing HTL results with the lattice calculations, one can conclude, that the $O\left(g^{6}\right)$ term is basically important for $T \leq 0.5 \mathrm{GeV}$ (see e.g. Fig. 1 of [6]). As a result a new HTL version appeared in [24], called "the $O\left(g^{6}\right)$ fitted" HTL contribution, as well as "the 
$O\left(g^{6}\right)$ fitted + nonpert." version. As we shall show below, the $O\left(g^{6}\right)$ terms indeed contain the whole series $O\left(g^{n}\right), n>6$, as was shown by Linde [1], but in addition the colormagnetic confinement makes these terms finite and summable. All this makes our analysis and discussion of the Linde problems even more timely and relevant.

The paper is organized as follows. In the next section we describe qualitatively the possible solution of the problem, in section 3 we write the general background field formalism for the thermodynamic potential, and define its perturbation series and we study the gluonic multiloop diagram with spatial (magnetic) confinement. We define its infrared and ultraviolet properties, showing that indeed the presence of $\sigma_{s}$ prevents the IR divergence of any diagram.

Section 4 is devoted to the summary and prospectives.

In appendix a detailed derivation is given of $\sigma_{s}$ in terms of gluon propagators and gluelumps.

\section{Qualitative analysis of the perturbative diagrams}

Coming back to the Linde problem (1), the standard perturbation theory (without nonperturbative background), which proceeds essentially in 3d, becomes infrared divergent, starting with the 6-th order in $g$ [1, 2]. In essence, the problem occurs due to very weak fall-off of the gluon propagator in $3 \mathrm{~d}$ without $\sigma_{s}$, e.g. in the $x$-space

$$
G(x, y) \sim \frac{T}{\pi|\mathbf{x}-\mathbf{y}|}, \quad|\mathbf{x}-\mathbf{y}| \gg 1 / T
$$

Let us now consider a $n$-th order diagram of the thermal perturbation theory, an example of this diagram for $n=8$ is shown in Fig. 1. One can count the number of gluon propagators, Eq. (3), in the diagram: $N_{\text {prop }}=\frac{3 n}{2}$.

The number of vertices with derivatives $\frac{\partial}{\partial x_{i}}$ at each vertex is $n$, the number

of space integrals $\frac{d^{3} x^{(i)}}{T}$ in each vertex is $n$, however one integral yields the overall volume, so that the amplitude can be written as

$$
A_{n} \sim g^{n} \prod_{i=1}^{n} \int \frac{d^{3} x^{(i)}}{T} \frac{\partial}{\partial x^{(i)}} \prod_{k=1}^{N_{\text {prop }}} G^{(k)}\left(x^{(i)}-x^{(j)}\right) \sim \frac{V_{3}}{T} \bar{A}_{n} .
$$

As a result one obtains the spatial dimension of the amplitude $\bar{A}_{n}$ in terms of an overall upper limit of $3 \mathrm{~d}$ coordinate $X$,

$$
\bar{A}_{n}=g^{n} X^{\frac{n}{2}-3} T^{\frac{n}{2}+1} .
$$


It is clear from (5), that $\bar{A}_{n}$ is IR divergent for $n \geq 6$, in agreement with the Linde problem 1 [1].

Now let us take into account the spatial (colormagnetic), confinement in $3 \mathrm{~d}$, which can be introduced in (4) in the form of the area law factor $\langle W(C)\rangle=$ $\exp \left(-\sigma_{s} S_{\min }\right)$, as in (1) with the minimal surface $S_{\min }=A_{3 d}(C)$, covering all diagram in Fig. 1. For us it is only important, that $S_{\min }$ is quadratic in coordinates $x^{(i)}, x^{(j)}$, and consequently it behaves at large $X$ as $S_{\min } \sim X^{2}$. Being positive definite, it makes the $\langle W(C)\rangle$ the real cut-off function, as it was proposed in [25], and can make the spatial integrals to converge, namely

$$
\bar{A}_{n}^{(\text {conf })}=g^{n} T^{\frac{n}{2}+1} \int(d X)^{\frac{n}{2}-3} \exp \left(-\sigma_{s}|X|^{2}\right) .
$$

It is clear, that introducing the dimensionless coordinate $Y=\sqrt{\sigma_{s}} X$, one obtains the following representation for $\bar{A}_{n}^{\text {(conf) }}$.

$$
\bar{A}_{n}^{\text {(conf) }}=g^{n} T^{\frac{n}{2}+1}\left(\sqrt{\sigma_{s}}\right)^{-\left(\frac{n}{2}-3\right)} J_{n}
$$

where $J_{n}$ is a dimensionless converging integral.

Now taking into account Eq. (2),$\sqrt{\sigma_{s}} \sim g^{2}(T) T$, one obtains finally

$$
\bar{A}_{n}^{\text {(conf) }}=g^{6} T^{4} C_{n}, \quad C_{n}=J_{n}\left(c_{\sigma}\right)^{3-\frac{n}{2}}
$$

Eq. (8) exemplifies the second part of the Linde problem: all the series with $n \geq 6$ contributes to the $O\left(g^{6}\right)$ term.

Note, that we have not introduced above the magnetic or any other mass parameters for gluons, since in the case of confinement the notion of mass can be ascribed only to the given string state, containing two (or more) gluons, connected by the adjoint string.

Nevertheless, if we introduce the effective mass of the gluon, $m_{\operatorname{mag}}(T)$, then the gluon Green's function acquires an additional factor $\exp \left(-m_{\operatorname{mag}}|\mathbf{x}-\mathbf{y}|\right)$, and these factors can be assembled in the total factor $\exp \left(-m_{\operatorname{mag}} \sum_{i, j}\left|\mathbf{x}_{i}-\mathbf{x}_{j}\right|\right)$, which would replace $\exp \left(-\sigma_{s}|X|^{2}\right)$ in (6) .

As a result one obtains instead of (7) the representation

$$
\bar{A}_{n}^{\text {(mass) }}=g^{2} T^{\frac{n}{2}+1}\left(m_{\mathrm{mag}}\right)^{-\left(\frac{n}{2}-3\right)} J_{n}^{(\mathrm{mass})},
$$

and assuming for $m_{\text {mag }}$ the form of magnetic mass $m_{\text {mag }}=c_{m} g^{2} T$, one again comes to the result (8). In this way one obtains that both spatial confinement and magnetic mass yield the same qualitative result: the sum of all $g^{n}$ terms with $n \geq 6$ contributes 
to the $O\left(g^{6}\right)$ term, in agreement with the second problem of Linde [1], and in both cases the space integrals converge. In the next section we shall make our arguments more concise, developing a special representation for a 4-point (or 3-point) diagram with confinement taken into account.

\section{Background perturbation theory in magnetic confinement}

In this section we exploit the background perturbation theory, developed in [8, 9, to study soft and hard regimes of the internal integrations and to demonstrate the role, which is played in this process by the magnetic confinement. Since we are mostly interested in the high $T$ gluon contributions, we confine ourselves to the case of pure gluodynamics.

We split the gluonic field $A_{\mu}$ into nonperturbative (np) background $B_{\mu}$ and the perturbative part $a_{\mu}$

$$
A_{\mu}=B_{\mu}+a_{\mu}
$$

and the partition function $Z$ can be written as a double average, using 'tHooft identity [8, 9]

$$
Z \equiv<<\exp (-S(B+a))>_{a}>_{B}
$$

where the action $S$ contains the standard gluon, ghost and gauge fixing terms and in particular the triple vertices $a^{3}, a^{2} B$.

The inverse gluon propagator can be written as

$$
G^{-1}=-D^{2}(B)_{a b} \cdot \delta_{\mu \nu}-2 g F_{\mu \nu}^{c}(B) f^{a c b}
$$

where

$$
\left(D_{\lambda}\right)_{c a}=\partial_{\lambda} \delta_{c a}-i g T_{c a}^{b} B_{\lambda}^{b}
$$

In what follows we shall for simplicity neglect the gluon spin term - the last term on the r.h.s. of (12) (the latter gives a correction to spatial (magnetic) confinement), and then the gluon propagator can be written as

$$
\left(-D^{2}\right)_{x y}^{-1}=<x\left|\int_{0}^{\infty} d t e^{t D^{2}(B)}\right| y>=\int_{0}^{\infty} d t(D z)_{x y}^{w} e^{-K} \Phi(x, y)
$$

where

$$
K=\frac{1}{4} \int_{0}^{s} d \tau\left(\frac{d z_{\mu}}{d \tau}\right)^{2}, \quad \Phi(x, y)=P \exp i g \int_{y}^{x} B_{\mu} d z_{\mu}
$$


and a winding path measure is

$$
(D z)_{x y}^{w}=\lim _{N \rightarrow \infty} \prod_{m=1}^{N} \frac{d^{4} \zeta(m)}{(4 \pi \varepsilon)^{2}} \sum_{n=0, \pm 1, . .}^{\infty} \int \frac{d^{4} p}{(2 \pi)^{4}} e^{i p\left(\sum \zeta(m)-(x-y)-n \beta \delta_{\mu 4}\right)}
$$

In the free case, $B_{\mu} \equiv 0$, one obtains the gluon propagator

$$
G(x, y) \rightarrow\left(\partial^{2}\right)_{x y}^{-1}=\sum_{k=0, \pm 1, \ldots} \int \frac{T d^{3} p}{(2 \pi)^{3}} \frac{e^{-i \vec{p}(\vec{x}-\vec{y})-i 2 \pi k T\left(x_{4}-y_{4}\right)}}{\left(\vec{p}^{2}+(2 \pi k T)^{2}\right)}
$$

At large distances the zero mode $(k=0)$ yields the behavior shown in (3) and this is the origin of the IR divergence of higher order $g^{n}$ contributions to the free energy, as was shown in [1], while magnetic confinement, contained in $\Phi(x, y)$, cuts off all divergences, as will be demonstrated below.

One can easily find the lowest order (one loop) np contribution to the free energy

$$
F_{0}^{g l}(B)=T\left\{\frac{1}{2} \log \operatorname{det} G^{-1}-\log \operatorname{det}\left(-D^{2}(B)\right)\right\},
$$

which can be written as

$$
F_{0}^{g l}(B)=-T \int_{0}^{\infty} \frac{d s}{s} \xi(s) d^{4} x(D z)_{x x}^{w} e^{-K}\left\langle\operatorname{tr}_{a} \Phi(x, x)\right\rangle_{B}
$$

and finally for the pressure $P_{g l}=-\frac{1}{V_{3}}\left\langle F_{0}^{g l}(B)\right\rangle$,

$$
P_{g l}=\left(N_{c}^{2}-1\right) \int_{0}^{\infty} \frac{d s}{s} \sum_{n \neq 0} G^{(n)}(s)
$$

with

$$
G^{(n)}(s)=\int(D z)_{o n}^{w} e^{-K}\left\langle\operatorname{tr}_{a} \Phi(x, x)\right\rangle_{B}
$$

$\Phi(x, x)$ contains colorelectric fields $B_{4}(x)$, which produce Polyakov lines $L_{a d j}(T)[9]$, and in addition also colormagnetic fields, which are contained in the spatial Wilson loop, $\left\langle\operatorname{tr}_{a} \Phi_{s}\left(C_{n}\right)\right\rangle_{B} \equiv\left\langle W_{s}\left(C_{n}\right)\right\rangle$, which can be written in terms of field correlators [7], as an integral over minimal surface inside the loop $C$

$$
\left\langle W_{s}\left(C_{n}\right)\right\rangle=t r_{a}\left\langle\exp \left(i g \int_{C} A_{\mu} d z_{\mu}\right)\right\rangle=t r_{a}\left\langle\exp \left(i g \int d s_{\mu \nu} F_{\mu \nu}\right)\right\rangle
$$

and using the cumulant expansion [7, 11, 19] and dropping all cumulants except for quadratic, one has 


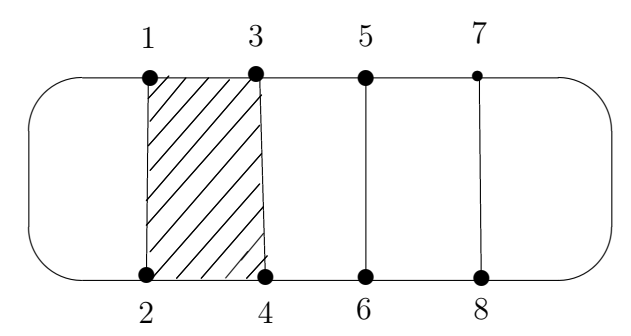

Figure 1: The 8-th order graph with the crossed rectangular under study

$$
\left\langle W_{s}\left(C_{n}\right)\right\rangle=\exp \left(-\frac{1}{2} \int_{S} \int_{S} d s_{\mu \nu}(u) d s_{\lambda \sigma}(v)\left\langle F_{\mu \nu}(u) F_{\lambda \sigma}(v)\right\rangle\right) .
$$

Considering only spatial loops $C$ and surface areas $S$ for $k=0$, i.e. the term without higher Matsubara frequencies, one has to do with colormagnetic correlators only,

$$
\frac{g^{2}}{N_{c}}\left\langle H_{i}(u) H_{j}(v)\right\rangle=\delta_{i j} D^{H}(u-v)+O\left(D_{1}^{H}\right) .
$$

To find $\sigma_{s}$ in (11) one can use the connection of $D^{H}$ with the gluelump Green's function [19], which, as shown in the Appendix, can be written as

$$
D^{H}(z)=\frac{g^{4}\left(N_{c}^{2}-1\right)}{2} T^{2} G_{3 d}^{(2 g)}(z)
$$

where $G_{3 d}^{(2 g)}$ is the two-gluon np Green's function in 3d. As a result using (11) one can write the $T$-dependent part of $\sigma_{s}$ as

$$
\sigma_{s}(T)=g^{4} T^{2} c_{\sigma}^{2}, \quad c_{\sigma}^{2}=\frac{N_{c}^{2}-1}{4} \int d^{2} z G_{3 d}^{(2 g)}(z),
$$

where $c_{\sigma}$ is dimensionless number and a fully np quantity.

Insertion of $\left\langle\operatorname{tr}_{a} \Phi\right\rangle$ in (21) as an area law (11) yields a loop graph of a gluon, where the string tension $\sigma_{s}$ controls the area inside the loop, so that the gluon cannot go far from the initial point, the maximal distance being $R \lesssim \frac{1}{\sqrt{\sigma_{s}}}$. One can now generalize this picture to the higher terms in the perturbative series $O\left(g^{n}\right)$, where these terms are formed by applying the term $L_{3}$ in the original QCD Lagrangian

$$
L_{3}=g \partial_{\mu} a_{\nu}^{a} f^{a b c} a_{\mu}^{b} a_{\nu}^{c}
$$


on any gluon line. As a result one obtains e.g. the diagram of Fig. 1 of the order $g^{8}$. It is essential, that each gluon propagator $G_{\mu \nu}^{a}\left(x^{(i)}, x^{(k)}\right) \equiv\left\langle a_{\mu}^{a}\left(x^{(i)}\right) a_{\nu}^{b}\left(x^{(k)}\right)\right\rangle$ is proportional to $\Phi\left(x^{(i)}, x^{(k)}\right)$ and the latter after averaging over background fields $B_{\mu}$, in the product together with all other gluon propagators, forms the total Wilson loop with the same outer contour $C_{n}$, but now with inner lines, dissecting it into a sum of pieces of area $\Delta A^{(i)}, \mathcal{A} \rightarrow \sum_{i} \Delta \mathcal{A}^{(i)}$, each piece is subject to the area law with the same $\sigma_{s}$, so that one obtains the factor $\exp \left(-\sigma_{s} \sum_{i} \Delta \mathcal{A}^{(i)}\right)$, which prevents the escape of all gluons from the center of the area, and in this way ensures infrared stability.

One can say, that each gluon is interacting with the closest neighbor via linear confining interaction and therefore the distance between them is of the order of $\left(\sqrt{\sigma_{s}}\right)^{-1}$.

We now turn to the more formal procedure to define the properties of the oneloop part of the complicated diagrams, shown in Fig.1, as a crossed rectangular. At each vertex of this diagram enters the operator (27), which generates $3 \mathrm{~g}$ vertex $\Gamma_{i}$ with momentum operator $p_{i}$, so that the quadratic loop diagram in the $3 \mathrm{~d}$ space can be written as

$$
G\left(p^{(1)}, p^{(2)}, p^{(3)}, p^{(4)}\right)=\prod_{i=1}^{4} \Gamma_{i} \int_{0}^{\infty} d s_{i}\left(D z^{(i)}\right)_{x^{(i)}, x^{(i-1)}} e^{-K_{i}} \Phi^{(i)} e^{i p^{(i)} x^{(i)}} d x^{(i)} .
$$

Here we have introduced the phase factors

$$
\Phi^{(i)}\left(x^{(i)}, x^{(i-1)}\right)=P_{A} \exp \left(i g \int_{x^{(i-1)}}^{x^{(i)}} A_{\mu} d z_{\mu}\right)
$$

omitting for simplicity the gluon spin phase factor, originating from the last term in (24), since it is inessential in the asymptotic limit for large $\left|x^{(i)}-x^{(i-1)}\right|$. Here $\left(D z^{(i)}\right)$ is

$$
\left(D z^{(i)}\right)_{x y}=\lim _{N \rightarrow \infty} \prod_{k=1}^{N} \frac{d^{3} \xi^{(i)}(k)}{(4 \pi \varepsilon)^{2}} \frac{d^{3} q^{(i)}}{(2 \pi)^{4}} e^{i q^{(i)}\left(\sum_{k} \xi^{(i)}(k)-(x-y)\right)}, \quad N \varepsilon=s .
$$

It is essential that the product of all phase factors $\Phi^{(i)}$ in the whole diagram of Fig. 1 should be averaged over vacuum configurations, yielding $3 \mathrm{~d}$ confinement, and each gluon line is in adjoint representation, and can be represented as the double fundamental line in the simple case of the large $N_{c}$ limit, so that one finally has a product of independent closed fundamental lines, circumvented by a common line 
in the outer contour. In the same large $N_{c}$ limit the average of this product can be represented as the product of averages of individual loops time the average of the outer contour, which yields the overall confining factor. In what follows we shall be interested in the properties of one rectangular loop and demonstrate its spatial convergence, while the overall confining loop will exhibit additional convergence.

The rather complicated calculations, given in the Appendix B of the paper [26] for the case $d=4$, can be done in an analogous manner for the case $d=3$, and one obtains the following form of the rectangular of Fig. 1 with account of the spatial confinement

$$
G_{4}\left(p_{i}\right)=(2 \pi)^{3} \delta^{(3)}\left(\sum p^{(i)}\right) \prod_{i=1}^{4} \int \frac{d^{3} q_{i} \Gamma_{i}}{q_{i}^{2}} I_{4}(b)
$$

where

$$
I_{4}(b)=\int \frac{d^{3} \mathcal{P}}{(2 \pi)^{3}}\left(\frac{4 \pi}{\sigma}\right)^{6} \exp \left(-\frac{2}{\sigma} \sqrt{b_{1}^{2} b_{2}^{2}-\left(\mathbf{b}_{1} \mathbf{b}_{2}\right)^{2}}\right) \exp \left(-\frac{2}{\sigma} \sqrt{b_{3}^{2} b_{4}^{2}-\left(\mathbf{b}_{3} \mathbf{b}_{4}\right)^{2}}\right),
$$

and $b_{i}$ are

$$
\begin{gathered}
b_{1}=q_{1}-p_{2}-p_{3}+\mathcal{P}, \quad b_{2}=q_{2}-p_{3}+\mathcal{P}, \\
b_{3}=q_{3}+\mathcal{P}, \quad b_{4}=q_{4}+p_{4}+\mathcal{P} .
\end{gathered}
$$

One can check, that at large momenta, (the hard regime) when $b_{i}^{2} \gg \sigma, i=$ $1,2,3,4$.

$$
I_{4}(b) \rightarrow \prod_{i=1,2,4} \delta^{(3)}\left(b_{i}\right)
$$

and the product of four factors $d^{3} q_{i}$ is reduced to a single integration $d^{3} q_{3}$, as it should be in the free case without confinement.

As a result one has in (31) for one loop in Fig. 1 the combination $d^{3} q \prod_{i=1}^{4} \frac{\Gamma_{i}}{q_{i}^{2}}$, and for the whole chain of $n$ loops, as in Fig. 1, one obtains an estimate (see [1])

$$
\mathcal{M}_{n}(T) \sim g^{2(n-1)}\left(T \int_{a \sqrt{\sigma}}^{T} d^{3} q\right)^{n} \frac{q^{2(n-1)}}{\left(q^{2}\right)^{3(n-1)}} .
$$

Here we have used the hard limit condition, $q \geq a \sqrt{\sigma}, a \gg 1$.

Integration in (35) yields the result $(n>4)$

$$
\mathcal{M}_{n}^{\mathrm{hard}}(T) \sim \frac{g^{2(n-1)} T^{n} \sigma_{s}^{\frac{4-n}{2}}}{a^{n-4}} \sim \frac{g^{6} T^{4}}{\left(c_{\sigma} a\right)^{n-4}}
$$


where we have exploited (2). This, apart from the $c_{\sigma} a$ factor, is the problem (2) of Linde [1]: all terms with $n>4$ contribute to the order $g^{6} T^{4}$, however with decreasing magnitude for $c_{\sigma} a \gg 1$.

To complete our study we consider now the soft regime, all momenta $q_{i}, p_{i}$ in (33) are small, $q_{i}, P \lesssim \sqrt{\sigma}$. In this case every loop integration $d^{3} q$ in (35) is replaced by

$$
d^{3} q \rightarrow \prod_{i=1}^{4} d^{3} q_{i} I_{4}(b)=\sigma_{s}^{3 / 2} f\left(\frac{q_{i}}{\sqrt{\sigma}}\right),
$$

where $f$ in (32) yields a cut-off in the $d^{3} q$ integration in (35), and as a result one obtains in the soft regime

$$
\mathcal{M}_{n}^{\mathrm{soft}}(T) \sim g^{2(n-1)} T^{n} \sigma_{s}^{\frac{4-n}{2}} \varphi_{n} \sim g^{6} T^{4} \varphi_{n}
$$

where $\varphi_{n}$ is a converging integral of dimensionless ratios $q_{i} / \sqrt{\sigma}$. One can see, that (38) yields qualitatively the same result as in (36), for the order of magnitude estimates. However quantitatively one should calculate nonperturbatively the whole series $n \geq 4$ to recover the $O\left(g^{6}\right)$ contribution. This situation is similar to the solution of the relativistic problem of two potentials: one confining and another gluon exchange but without small parameters, and one should sum up the series, or rather solve the corresponding relativistic Hamiltonian equation [27].

\section{Summary and prospectives}

We have considered above the gluon thermodynamics with the nonperturbative background fields, which ensure spatial confinement due to colormagnetic correlators (24). As a result one obtains the area law of the spatial Wilson loop with the nonzero spatial string tension. Qualitatively it is clear, that all multigluon diagrams in $3 \mathrm{~d}$ would be convergent at large spatial distances, and this property was used in [25] to argue that the Linde problem is absent in the confining vacuum. In the present paper this qualitative argument was given a more quantitative foundation.

Indeed, the explicit account of the spatial confinement not only formally solves the Linde problem, but it is also vitally important in the thermodynamics of the quark gluon plasma (qgp), as was shown recently in the case of the $\mathrm{SU}(3)$ [28, 29], as well as in the case of $n_{f}=2+1$ thermodynamics in the deconfined phase [30]. It

was demonstrated there, that taking into account correlator $D_{1}^{E}$ (which generates 
Polyakov lines) and $D^{H}$ for spatial confinement one obtains a good agreement with most accurate lattice data.

As we argued above, qualitatively one can exploit the universal effective gluon mass $m_{D}^{H}(T) \cong 2 \sqrt{\sigma_{s}(T)}$ instead of spatial confinement with $\sigma_{s}(T)$ as a first approximation in the effective perturbation theory up to the $g^{6}$ order.

From this point of view we have stressed the existence of the effective screening mass parameter, which is of np origin and occurs due to magnetic confinement string tension $\sigma_{s}$ - this is the answer to what we call the problem 1 of Linde. The second problem of Linde, the infinite $g^{n}$ series with $n \geq 6$, contributing to the order $g^{6} T^{4}$, is confirmed above in the np approach.

As was mentioned above, the whole dynamics of diagrams with $n \geq 6$ lies in the soft np region, where the magnetic confinement and not gluon exchange mechanism play the most important role. It is an open question what is the sum of $n>6 \mathrm{np}$ terms with magnetic confinement, which is equivalent to the $g g$ amplitude in the case of two interactions in $3 \mathrm{~d}$ : confining $V_{\text {conf }}$ and gluon exchange $V_{O G E}$, but the answer is possible to obtain.

At this point it is worthwhile to compare our results with other approaches, where the notion of confinement plays an important role. In the Gribov-Zwanziger (GZ) method (see e.g. [13, 14]) the local gluon mass is introduced, which at large $T$ has the same form as the magnetic mass $m_{\text {mag }}=c_{m} g^{2} T$ in (9), but with a different coefficient, and it it is stated in [14], that the resummation of the perturbation series with this mass yields results, consistent with lattice data.

It is not evident, that the whole procedure of the gluon mass generation is fully gauge invariant, however even assuming this, the simultaneous appearance of confinement and the generated mass of gluons (and the use of only the second factor) calls for additional inquiry.

Another question of the GZ approach is the possible difficulty of the vector confinement with the so-called Klein paradox, leading to the unboundedness of the fermion spectrum in the confining vector potential, see [31] for details.

Apart from this, the GZ approach suggests to solve the Linde problem 1 via magnetic gluon mass generation.

Following this line of IR problem resolution via confinement, it is worthwhile to mention the active development of the methods of compactified QCD, and in particular with respect to weak-coupling semiclassical realization of IR renormalons as in [34]. The problems of IR renormalons have been also studied in connection to the structure of singularities in the Borel plane in [32].

It is remarkable, how confinement operates also in this respect. In [33] it was 
shown, that confinement resolves the IR renormalon problem, transforming the series of renormalon loop graphs, covered with confining film, into the Borel summable series. In this way the whole perturbative series in the confining background can be treated within the standard renormalization technique without IR renormalon singularities.

In a similar way the well-known Landau ghost problem of the IR divergence of the renormalised $\alpha_{s}\left(Q^{2}\right)$ was solved in [35]. Summarizing, one can say, that confinement actually solves the known IR problem of QCD.

The author is grateful for useful discussions to M.A.Andreichikov, B.O.Kerbikov and M.S.Lukashov. This work was done in the framework of the scientific project, supported by the Russian Science Fund, grant 16-12-10414.

Appendix 1 Calculation of the spatial string tension via two-gluon Green's function

To calculate $D^{H}(z)$ one can use the technic, developed in [19] for $D^{E}(z)$, which allows to express it via two-gluon Green's function $G_{4 d}^{(2 g)}(z)=G_{4 d}^{(g)} \otimes G_{4 d}^{(g)}$, where two gluons interact nonperturbatively.

The starting point for the gluon propagator $G_{4 d}^{(g)}$ is the integration in the 4 th direction in (14) with the exponent $K_{4}=\frac{1}{4} \int_{0}^{s} d \tau\left(\frac{d z_{4}}{d \tau}\right)^{2}$, which gives for the spatial loop with $x_{4}=y_{4}$,

$$
J_{4} \equiv \int\left(D z_{4}\right)_{x_{4} x_{4}} e^{-K_{4}}=\sum_{n=0, \pm 1, \ldots} \frac{1}{2 \sqrt{\pi s}} e^{-\frac{(n \beta)^{2}}{4 s}}=\frac{1}{2 \sqrt{\pi s}}\left(1+\sum_{n= \pm 1, \pm 2} e^{-\frac{(n \beta)^{2}}{4 s}}\right) .
$$

The second term in (A1.1) at large $T \gg \frac{1}{2 \sqrt{s}}$ yields $2 \sqrt{\pi s} T$, which gives $J_{4}=$ $\frac{1}{2 \sqrt{\pi s}}+T$.

The same linear in $T$ term is obtained using the Poisson relation [8, 9]. As a result the $4 \mathrm{~d}$ gluon propagator is reduced to the $3 \mathrm{~d}$ one,

$$
G_{4 d}^{(g)}(z)=T G_{3 d}^{(g)}(z)+K_{3 d}(z)
$$

where $K_{3 d}(z)$ does not depend on $T$. In what follows we consider only the first term in (A1.2), keeping in mind, that $G_{4 d}^{(g)}(z)$ at small $T$ has a nonzero limit. Substituting 
this term in the general expression for $D^{E}(z)\left(D^{H}(z)\right)$ obtained in [19], one has

$$
D^{H}(z)=\frac{g^{4}\left(N_{c}^{2}-1\right)}{2}\left\langle G_{4 d}^{(2 g)}(z)\right\rangle \rightarrow \frac{g^{4}\left(N_{c}^{2}-1\right) T^{2}}{2}\left\langle G_{3 d}^{(2 g)}(z)\right\rangle,
$$

where $G_{3 d}^{(2 g)}$ is the two-gluon Green's function in 3d with all interaction between gluons taken into account

$$
\left\langle G_{3 d}^{(2 g)}\right\rangle=\left\langle G_{3 d}^{(g)}(x, y) G_{3 d}^{(g)}(x, y)\right\rangle_{B}
$$

In terms of the gluelump phenomenology, studied in [20, 21 (A1.4) is called the twogluon gluelump, computed on the lattice in [21] and analytically in [20]. In our case we are interested in the $3 \mathrm{~d}$ version of the corresponding Green's function. Choosing in $3 \mathrm{~d}$ the $x_{3} \equiv t$ axis as the Euclidean time, we proceed as in [16], exploiting the path integral technic [7, 26], which yields

$$
G_{3 d}^{(2 g)}(x-y)=\frac{t}{8 \pi} \int_{0}^{\infty} \frac{d \omega_{1}}{\omega_{1}^{3 / 2}} \int_{0}^{\infty} \frac{d \omega_{2}}{\omega_{2}^{3 / 2}}\left(D^{2} z_{1}\right)_{x y}\left(D^{2} z_{2}\right)_{x y} e^{-K_{1}\left(\omega_{1}\right)-K_{2}\left(\omega_{2}\right)-V t},
$$

where $V$ includes spatial confining interaction between the three objects: gluon 1, gluon 2 and the fixed straight line of the parallel transporter, which makes all construction gauge invariant (see [20, 19] for details). In (A1.5) $t=|x-y| \equiv|w|$; and finally

$$
\sigma_{s}(T)=\frac{g^{4}\left(N_{c}^{2}-1\right) T^{2}}{4} \int\left\langle G_{3 d}^{(2 g)}(w)\right\rangle d^{2} w .
$$

Constructing in the exponent of (A1.5) the 3 body Hamiltonian in the $2 \mathrm{~d}$ spatial coordinates

$$
H\left(\omega_{1}, \omega_{2}\right)=\frac{\omega_{1}^{2}+\mathbf{p}_{1}^{2}}{2 \omega_{1}}+\frac{\omega_{2}^{2}+\mathbf{p}_{2}^{2}}{2 \omega_{2}}+V\left(\mathbf{z}_{1}, \mathbf{z}_{2}\right),
$$

one can rewrite (A1.5) as follows, see [26]

$$
G_{3 d}^{(2 g)}(t)=\frac{t}{8 \pi} \int_{0}^{\infty} \frac{d \omega_{1}}{\omega_{1}^{3 / 2}} \int_{0}^{\infty} \frac{d \omega_{2}}{\omega_{2}^{3 / 2}} \sum_{n=0}^{\infty}\left|\psi_{n}(0,0)\right|^{2} e^{-M_{n}\left(\omega_{1}, \omega_{2}\right) t} .
$$

Here $\left.\Psi_{n}(0,0) \equiv \Psi_{n}\left(\mathbf{z}_{1}, \mathbf{z}_{2}\right)\right|_{\mathbf{z}_{1}=\mathbf{z}_{2}=0}$, and $M_{n}$ is the eigenvalue of $H\left(\omega_{1}, \omega_{2}\right)$. The latter was studied in [20] in 3 spatial coordinates. For our purpose here we only mention, that $G_{3 d}^{(2 g)}(z)$ has the dimension of the mass squared and the integral in (A1.6) is therefore dimensionless. Hence one obtains $\sqrt{\sigma_{s}(T)}=g^{2} T c_{\sigma}+$ const, as was stated above in (3), where

$$
c_{\sigma}^{2}=\frac{\left(N_{c}^{2}-1\right)}{4} \int d^{2} w\left\langle G_{3 d}^{(2 g)}(w)\right\rangle
$$

and const is obtained from the second term in (A1.2). 


\section{References}

[1] A.D.Linde, Phys. Lett. B 96, 289 (1980); Rep. Prog. Phys. 42, 389 (1979).

[2] D.J.Gross, R.D.Pisarski and L.G.Yaffe, Rev. Mod Phys. 53, 43 (1981).

[3] E.Braaten, R.D.Pisarski, Mucl. Phys. B 337, 569 (1990); J.O.Andersen, E.Braaten, M.Strickland, Phys. Rev. Lett. 83, 2139 (1999); J.O.Andersen, M.Strickland, Ann. Phys. 317, 281 (2005); U.Kraemmer, A.Rebhan, Rept. Prog. Phys. 67, 351 (2004).

[4] J. O. Andersen, L. E. Leganger, M. Strickland, and N. Su, JHEP 08, 053 (2011); S. Mogliacci, J. O. Andersen, M. Strickland, N. Su, and A. Vuorinen, JHEP 12, 055 (2013); N. Haque, J. O. Andersen, M. G. Mustafa, M. Strickland, and N. Su, Phys. Rev. D 89, 061701 (2014); N. Haque, A. Bandyopadhyay, J. O. Andersen, M. G. Mustafa, M. Strickland, and N. Su, JHEP 05, 027 (2014).

[5] Nan Su, Int. J.Mod. Phys., A 30, 1530025 (2015); arXiv:1502.04589.

[6] K.Kajantie, M.Laine, K.Rummukainen and Y.Schröder, Phys. Rev. D 67, 105008 (2003); hep-ph/0211321.

[7] H.G.Dosch, Phys. Lett. B 190177 (1987); H.G.Dosch, Yu.A.Simonov, Phys. Lett. B 205339 (1988); Yu.A.Simonov, Nucl. Phys. B 307512 (1988).

[8] Yu.A.Simonov, JETP Lett. 54, 249 (1991), ibid. 55, 627 (1992); Yu.A.Simonov, Phys. At. Nucl. 58, 309 (1995) [hep-ph/9311216]; N.O.Agasian, JETP Lett. 57, 208 (1993); ibid. 71, 43 (2000); H.G.Dosch, H.J.Pirner, Yu.A.Simonov, Phys. Lett. B349 335 (1995).

[9] Yu.A.Simonov, Ann. Phys. 323, 783 (2008); E.V.Komarov, Yu.A.Simonov, Ann. Phys. 323, 1230 (2008); Yu.A.Simonov, M.A.Trusov, JETP Lett. 85, 730 (2007); Phys. Lett. B 650, 36 (2007).

[10] Yu.A.Simonov, Phys. Usp. 39 , 313 (1996).

[11] A.Di Giacomo, H.G.Dosch, V.I.Shevchenko and Yu.A.Simonov, Phys. Rept. 372, 319 (2002).

[12] A.V.Nefediev, Yu.A.Simonov and M.A.Trusov, Int. J. Mod. Phys. E 18, 549 (2009). 
[13] D.Zwanziger, Phys. Rev. D 76, 125014 (2007), hep-ph/0610021;

K.Lichtenegger, D.Zwanziger, Phys. Rev. D 78, 034038 (2008), arXiv:0805.3804.

[14] K.Fukushima and Nan Su, Phys. Rev. D 88, 076088 (2008), arXiv:1304.8004; Nan Su and K.Tywoniuk, Phys. Rev. Lett. 114, 161601 (2015), arXiv:1409.3203.

[15] A.Di Giacomo, E.Meggiolaro and H.Panagopoulos, Nucl. Phys. B 483371 (1997);

M.D'Elia, A.Di Giacomo and E.Meggiolaro, Phys. Lett. D 67, 114504 (2003).

[16] E.L.Gubankova, Yu.A.Simonov, Phys. Lett. 360, 93 (1995).

[17] V.Koch, E.V.Shuryak, G.E.Born, and A.D.Jackson, Phys. Rev. D 46, 3169 (1992); C.Bernard et.al. Phys. Lett. 68, 2125 (1992).

[18] A.V.Nefediev, Yu.A.Simonov, Phys. At. Nucl. 71, 171 (2008).

[19] Yu.A.Simonov, Phys. At. Nucl. 69, 528 (2006);

Yu.A.Simonov, V.I.Shevchenko, Adv. High Energy Phys. 2009, 873051 (2009).

[20] Yu.A.Simonov, Nucl. Phys. B 592, 350 (2001).

[21] I.Jorysz, C.Michael, Nucl. Phys. B 302, 448 (1988);

N.Campbell, I.Jorysz and C.Michael, Phys. Lett. B 167, 91 (1986).

[22] G.Boyd, O.Kaczmarek and F.Zantow, Nucl. Phys. B 469, 419 (1996); hep-lat/0512031;

F.Karsch, E.Laermann, and M.Lütgemeier, Phys. Lett. B 346, 94 (1995).

[23] L.McLerran, Rev. Mod. Phys. 58, 1021 (1986); J.Kapusta, Finite Temperatute Field Theory (Cambridge University Press, Cambridge, 1989).

[24] Sz.Borsanyi, G.Endrodi, Z.Fodor, S.D.Katz and K.K.Szabo, JHEP 07,056 (2012), arXiv: 1204.6184.

[25] Yu.A.Simonov, in "Varenna 1995, Selected Topics in Nonperturbative QCD", p.319, 1995; hep-ph/9509404.

[26] Yu.A.Simonov, Int. J. Mod. Phys. A 31, 1650016 (2016). 
[27] Yu.A.Simonov, Phys. Rev. D 88, 025028 (2013).

[28] N.O.Agasian, M.S.Lukashov and Yu.A.Simonov, Mod. Phys. Lett. A 31, 1650222 (2016); arXiv: 1610.01472 [hep-ph].

[29] N.O.Agasian, M.S.Lukashov and Yu.A.Simonov, arXiv: 1701.07959.

[30] M.S.Lukashov and Yu.A.Simonov, arXiv: 1703.06666.

[31] J.D.Bjorken and S.D.Drell, Relativistic Quantum Mechanics, Mc Graw Hill, NY, (1964) pp.40-43; V.D.Mur, V.S.Popov, Yu.A.Simonov and V.P.Yurov, JETP 78, 1 (1994).

[32] G.V.Dunne and M.Üsal, Ann. Rev. Nucl. Part. Sci. 66, 245 (2016); arXiv: 1601.03414.

[33] G.V.Dunne, M.A.Shifman and M.Üsal, Phys. Rev. Lett. 114, 191601 (2015); arXiv:1502.06680.

[34] Yu.A.Simonov, JETP Lett. 57, 525 (1993).

[35] Yu.A.Simonov, Phys. At. Nucl. 74, 1223 (2011), arXiv:1011.5386. 